\title{
Characterizing natural fractures productivity in tight gas reservoirs
}

\author{
Hassan Bahrami $\cdot$ Reza Rezaee $\cdot$ Mofazzal Hossain
}

Received: 17 March 2012/Accepted: 25 June 2012/Published online: 10 July 2012

(C) The Author(s) 2012. This article is published with open access at Springerlink.com

\begin{abstract}
Tight formations normally have production problems mainly due to very low matrix permeability and various forms of formation damage that occur during drilling completion and production operation. In naturally fractured tight gas reservoirs, gas is mainly stored in the rock matrix with very low permeability, and the natural fractures have the main contribution on total gas production. Therefore, identifying natural fractures characteristics in the tight formations is essential for well productivity evaluations. Well testing and logging are the common tools employed to evaluate well productivity. Use of image log can provide fracture static parameters, and welltest analysis can provide data related to reservoir dynamic parameters. However, due to the low matrix permeability and complexity of the formation in naturally fractured tight gas reservoirs, welltest data are affected by long wellbore storage effect that masks the reservoir response to pressure change, and it may fail to provide dual-porosity dual-permeability models dynamic characteristics such as fracture permeability, fracture storativity ratio and interporosity flow coefficient. Therefore, application of welltest and image log data in naturally fractured tight gas reservoirs for
\end{abstract}

H. Bahrami $(\bowtie) \cdot$ R. Rezaee $\cdot$ M. Hossain

Department of Petroleum Engineering, Curtin University,

Level 6, ARRC, 26 Dick Perry Ave, Kensington, Perth 6151,

Australia

e-mail: hassan.bahrami@postgrad.curtin.edu.au

R. Rezaee

e-mail: R.Rezaee@curtin.edu.au

M. Hossain

e-mail: md.hossain@curtin.edu.au meaningful results may not be well understood and the data may be difficult to interpret. This paper presents the estimation of fracture permeability in naturally fractured tight gas formations, by integration of welltest analysis results and image log data based on Kazemi's simplified model. Reservoir simulation of dual-porosity and dual-permeability systems and sensitivity analysis are performed for different matrix and fracture parameters to understand the relationship between natural fractures parameters with welltest permeability. The simulation results confirmed reliability of the proposed correlation for fracture permeability estimation. A field example is also shown to demonstrate application of welltest analysis and image log data processing results in estimating average permeability of natural fractures for the tight gas reservoir.

Keywords Fracture permeability - Tight gas reservoirs . Natural fractures characterisation - Welltest analysis

$\begin{array}{ll}\text { List of symbols } \\ P & \text { Pressure } \\ K & \text { (Perm) Permeability } \\ Q & \text { Flow rate } \\ C & \text { Compressibility } \\ t & \text { Time } \\ h & \text { Layer thickness } \\ r & \text { Radius } \\ \varphi & \text { (Poro) Porosity } \\ a & \text { Fracture spacing } \\ b & \text { Fracture aperture } \\ \delta & \text { Shape factor } \\ \lambda & \text { Interporosity flow coefficient } \\ \omega & \text { Fracture storativity } \\ \mu & \text { Viscosity } \\ B & \text { Formation volume factor }\end{array}$

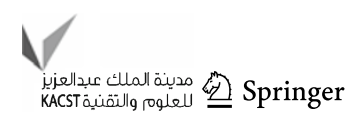


NFR Naturally fractured reservoirs

TGR Tight gas reservoirs

\section{Subscripts \\ $f \quad$ Fracture \\ $m$ Matrix}

\section{Introduction}

A naturally fractured reservoir is mainly a network of natural fractures and matrix which are randomly distributed. Characterization of the natural fractures generally includes estimating the dynamic parameters such as fracture permeability, and determining the static parameters such as fracture spacing (matrix block size), fracture aperture and fracture porosity (Racht and Golf 1982).

The most common geometrical representations of fractured reservoirs are the models introduced by Warren-Root and Kazemi as shown in Fig. 1, assuming that discrete matrix blocks are separated by an orthogonal system of continuous and uniform fractures. The matrix blocks are assumed to be isotropic and homogeneous identical rectangular parallelepipeds with no direct communication between them (Kazemi et al. 1976). The simplified models have been introduced to simulate flow through naturally fractured reservoirs. The double porosity domain assumes a continuous uniform fracture network oriented parallel to the principal axes of permeability.

In many of the naturally fractured reservoirs, fracture permeability can be the major controlling factor of the flow of fluids. Fracture permeability in a dual-porosity and dualpermeability reservoir is the permeability that is associated with the secondary porosity created by open natural fractures (Racht and Golf 1982). The main dynamic parameters commonly used to describe matrix and interconnecting fracture network are interporosity flow coefficient $(\lambda)$ and fracture storativity ratio $(\omega)$ that are defined as follows (Tiab et al. 2006):

$\lambda=\delta \frac{K_{m}}{K_{f}} r_{w}^{2}$

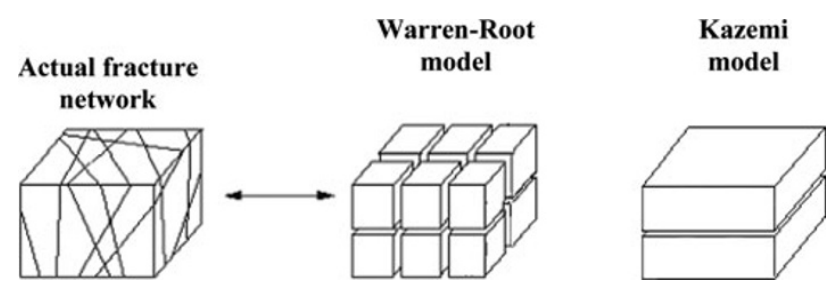

Fig. 1 Dual porosity-dual permeability system (Warren-Root and Kazemi simplified models) $\omega=\frac{\varphi_{f} \cdot C_{f}}{\varphi_{f} \cdot C_{f}+\varphi_{m} \cdot C_{m}}$

Where $K_{m}$ is matrix permeability, $K_{f}$ is fracture permeability, $r_{w}$ is wellbore radius, $\varphi_{f}$ is fracture porosity, $\varphi_{m}$ is matrix porosity, $C_{f}$ is fracture compressibility, $C_{m}$ is matrix compressibility, and $\delta$ is shape factor and it is defined as follows:

$\delta=4\left(1 / a_{X}^{2}+1 / a_{Y}^{2}+1 / a_{Z}^{2}\right)$

In Eq. (3), $a_{x}, a_{y}$ and $a_{z}$ are matrix block size respectively in $x, y$ and $z$ directions (Reiss 1980). In the case of Kazemi model $\left(a_{x} \gg a_{z}\right.$ and $\left.a_{y} \gg a_{z}\right)$, the shape factor, $\delta$, is considered to be $4 / a^{2}$. The smaller value of $\lambda$ (higher fracture permeability) and/or the larger value of $\omega$ (higher fracture porosity) result in higher well productivity.

The dual-porosity and dual-permeability reservoirs' dynamic parameters can be estimated using welltest analysis. As illustrated in Fig. 2, a Semi-Log plot of pressure build-up data results in two parallel lines, which the slope gives average permeability, the vertical separation between the parallel lines $\left(\Delta \mathrm{P}_{\omega}\right)$ can provide fracture storativity ratio, and the $\Delta \mathrm{P}$ at mid-point of the transition period $\left(\Delta \mathrm{P}_{\lambda}\right)$ can estimate interporosity flow coefficient (Saeidi Ali 1987).

The main input parameters required to model fluid flow through a naturally fractured formation are fracture permeability, fracture porosity and shape factor (Kazemi et al. 1976). The matrix block size (fracture spacing) to compute the shape factor is primarily obtained from borehole images. The fracture spacing can be attained using results of any type of borehole images (regardless of the drilling mud system used). However, in the case of water-based mud imaging (e.g., FMI), image log processing can also provide fracture aperture and porosity as an additional output of fracture analysis (Dashti and Bagheri 2009; Luthi 1990).

Integrating the welltest results with image log processing results for fracture spacing, and core data for matrix porosity, permeability and compressibility, can be used to estimate fracture permeability and fracture porosity from Eqs. (1) and (2) (Tiab et al. 2006):

$\varphi_{f}=\varphi_{m} \frac{C_{m}}{C_{f}} \frac{\omega}{1-\omega}$

$K_{f}=\delta \frac{K_{m}}{\lambda} r_{w}^{2}$

Equations (4) and (5) can determine fracture permeability and fracture porosity, in the case that the dual-porosity response is clearly observed on pressure build-up diagnostic plots, and $\omega$ and $\lambda$ values can be estimated certainly. Fracture compressibility in the fracture porosity estimation [Eq. (4)] may have uncertainties (maybe 1-100 folds higher than matrix compressibility) and might be estimated using well testing (Tiab et al. 2006). 
Fig. 2 Pressure transient behavior in naturally fractured reservoirs

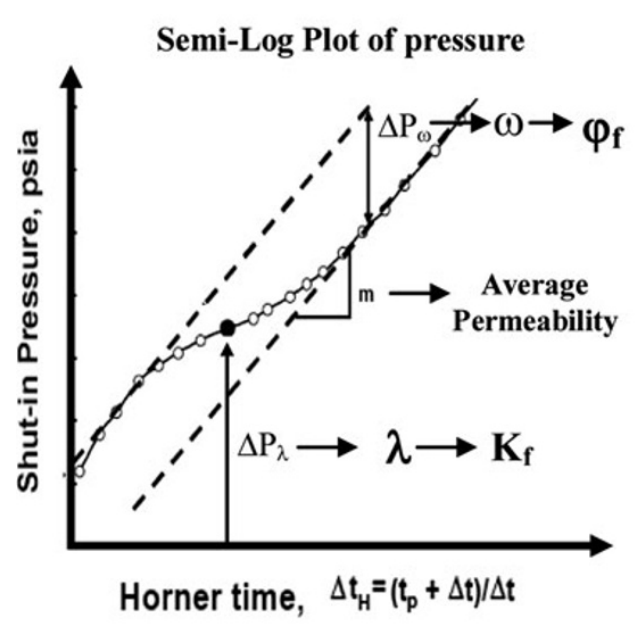

Log-Log Plot of pressure and pressure derivative

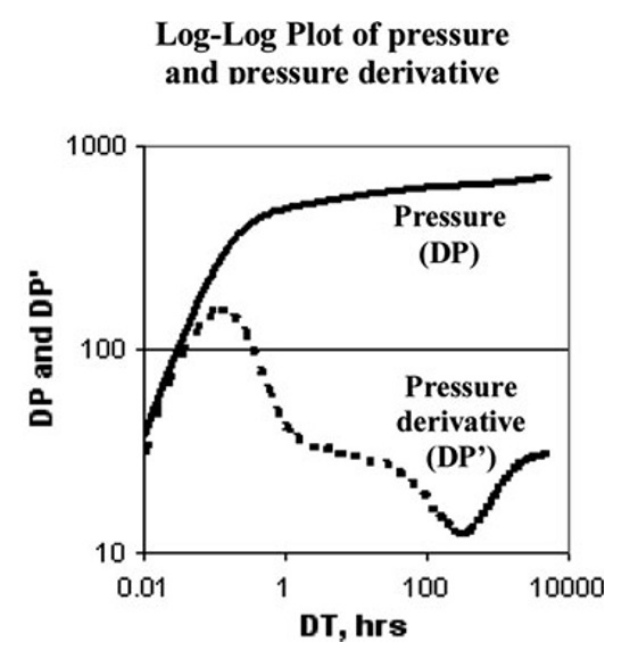

The image log porosity can be used to verify accuracy of average fracture porosity estimated from welltest analysis.

In tight reservoirs, the very low matrix permeability, complexity of the formation, and long wellbore storage effect may mask the reservoir response to the pressure change during transient testing. Although one can estimate the average permeability value from welltest data in tight reservoirs using advanced welltest interpretation techniques (Bahrami et al. 2010), estimating fracture storativity and interporosity flow coefficient from such welltest data might not be feasible since the dual-porosity response may not clearly be observed on pressure build-up diagnostic plots. The conventional approaches might fail to characterize fracture parameters in naturally fractured tight gas reservoirs, especially in complicated cases such as hydraulically fractured or horizontally drilled wells (Restrepo and Tiab 2009), and therefore application of welltest and image log data in the reservoirs may not be well understood and is proved to be difficult to interpret for meaningful results.

\section{Natural fractures characterisation in tight gas reservoirs}

Analysis of acquired data from a tight gas reservoir may provide limited information about the formation characteristics, due to some restrictions such as type of the drilling fluid, complicated and slow response of reservoir, not long enough testing time, etc. (Garcia et al. 2006). Hence, a simple model needs to be used that requires minimum data inputs in determining fracture parameters. The model introduced by Kazemi as shown in Fig. 3 can be used to build a simple dual-porosity and dual-permeability system of naturally fractured tight gas reservoirs. Considering Kazemi model that assumes parallel layers of matrix and fracture in a 


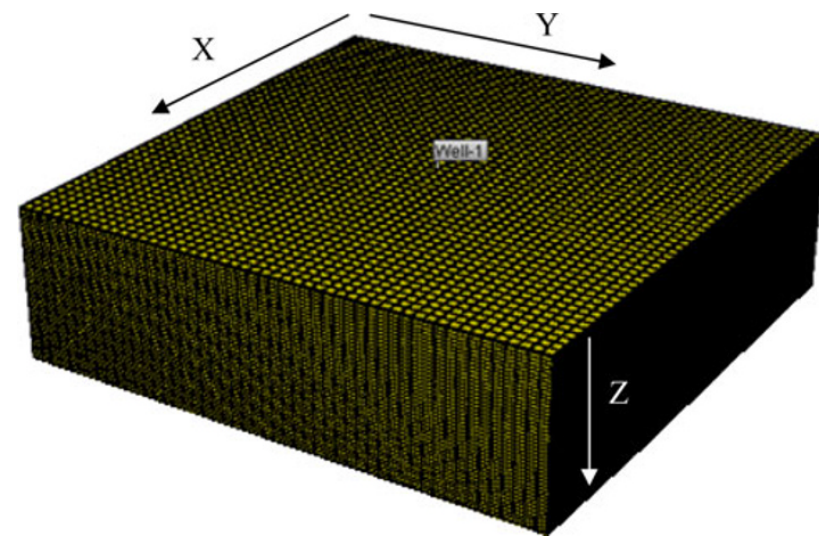

Fig. 4 Reservoir model 3D view, 50 grids in $X$ direction, 50 grids in $Y$ direction and 71 girds in $Z$ direction ( 36 horizontal matrix layers, 35 horizontal fracture layers)

Since Eq. (8) is based on simplified models and assumptions, using some correction factors might provide more realistic relationship between fracture dynamic parameters. Considering the correction factor, fracture permeability can be expressed in the following generalized form:

$K_{f}=C_{1} * K_{\text {welltest }} *\left(\frac{a}{b}\right)^{C_{2}}$

The constants $C_{1}$ and $C_{2}$ in Eq. (9) are the correction factors that need to be determined from numerical simulation and sensitivity analysis. Using the Eq. (9), natural fractures permeability can be estimated as function of average permeability $\times$ thickness (from welltest analysis) and average fracture spacing and aperture (from image log processing results). Once the natural fracture parameters are estimated, then using Eqs. (1) and (2) fracture storativity ratio and interporosity flow coefficient can be estimated for welltest design applications, well productivity evaluation, and gas production rate forecasting.

\section{Effect of natural fracture parameters on welltest response}

To evaluate natural fracture parameters in tight gas reservoirs, reservoir simulation is performed based on the field data from a tight gas reservoir, using the widely used commercial CMG (Computer Modeling Group of Calgary) numerical reservoir simulation software. The model is fully implicit in its basic formulation, and the nonlinear equations in the software are solved by Newtonian iteration with the derivatives of the Jacobian matrix evaluated numerically (Odeh and Aziz 1981).

Reservoir simulation model for dual-porosity and dualpermeability systems is developed by considering matrix layers that have been separated by fracture layers as described in Fig. 3. A well is considered at the center of the model, which has been completed in all the matrix and fracture layers with no flow boundary. The reservoir model has been shown in Fig. 4 and the input data used in the reservoir simulation are provided in Table 1.

Different simulation models are run with different fracture parameters to analyse sensitivity of pressure buildup response outputs to each fracture parameter. The sensitivity analysis is performed for different matrix and fracture parameters to understand the relationship between natural fractures static and dynamic parameters. The simulation model scenarios are provided in Table 2. Each simulation run consists of a production period with gas production rate of $500 \mathrm{MSCFD}$, followed by pressure build-up period. The pressure build-up data are analysed to estimate welltest permeability for each dual-porosity dual permeability system, and then determine the relationship between each fracture parameter and welltest analysis results.

First, the model is run for different fracture aperture values of $0.1,1$ and $10 \mathrm{~mm}$. Analysis of pressure drawdown data from the simulation runs is shown in Fig. 5. The early time data are affected by wellbore storage effect and

Table 1 Input data to the simulation base model

\begin{tabular}{llllll}
\hline No of grids in $X$ direction & 50 & - & Matrix compressibility & $4 \mathrm{E}-06$ \\
No of grids in $Y$ direction & 50 & - & Fracture compressibility & $4 \mathrm{E}-06$ & $1 / \mathrm{psia}$ \\
No of matrix layers & 36 & - & Fracture layer porosity & 100 & $\%$ \\
No of fracture layers & 35 & - & Matrix layer porosity & 8 \\
Grid size in $X$ direction & 70 & $\mathrm{ft}$ & Gas S.G. & 0.65 & - \\
Grid size in $Y$ direction & 70 & $\mathrm{ft}$ & Reservoir pressure & 3000 & 180 \\
Fracture layer thickness & 1 & $\mathrm{~mm}$ & Reservoir temperature & 180 & $\mathrm{~F}$ \\
Fracture spacing & 5 & $\mathrm{ft}$ & Net thickness & 0.25 & $\mathrm{ft}$ \\
Matrix permeability & 0.1 & $\mathrm{md}$ & Wellbore radius & Gas production rate & 500 \\
Fracture permeability & 50000 & & & MSCFD \\
\hline
\end{tabular}


Table 2 Input data to the simulation base model

\begin{tabular}{lll}
\hline Sensitivity analysis simulation scenarios & \\
\hline Fracture aperture & $\mathrm{mm}$ & $0.1,1,10$ \\
Fracture spacing & $\mathrm{ft}$ & $5,10,20$ \\
Permeability of fracture layer & Darcies & $10,50,100$ \\
Porosity of fracture layer & fraction & $0.6,0.8,1$ \\
Matrix compressibility & $1 / \mathrm{psia}$ & $4 \mathrm{E}-5,4 \mathrm{E}-6,4 \mathrm{E}-7$ \\
Fracture compressibility & $1 / \mathrm{psia}$ & $4 \mathrm{E}-5,4 \mathrm{E}-6,4 \mathrm{E}-7$ \\
Matrix permeability & $\mathrm{md}$ & $0.005,0.1,2$ \\
\hline
\end{tabular}

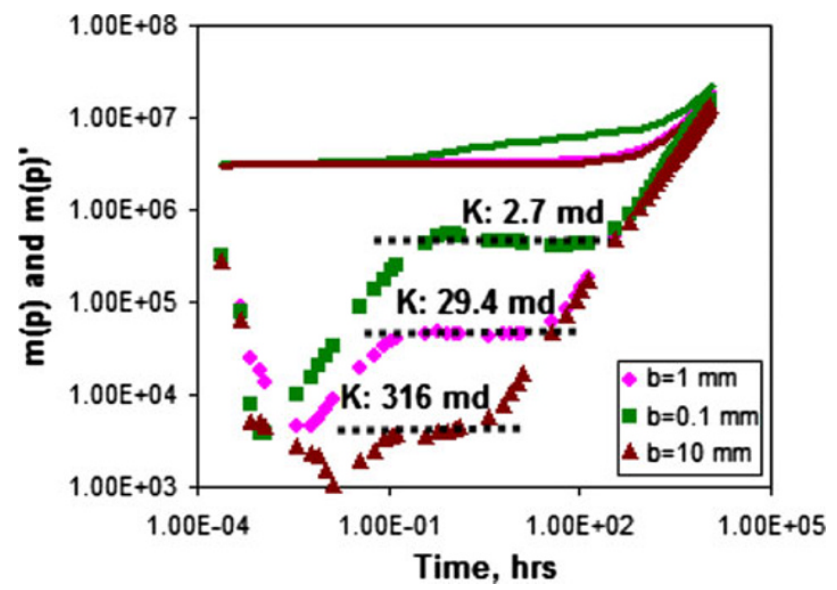

Fig. 5 Effect of fracture aperture $(b)$ on welltest permeability

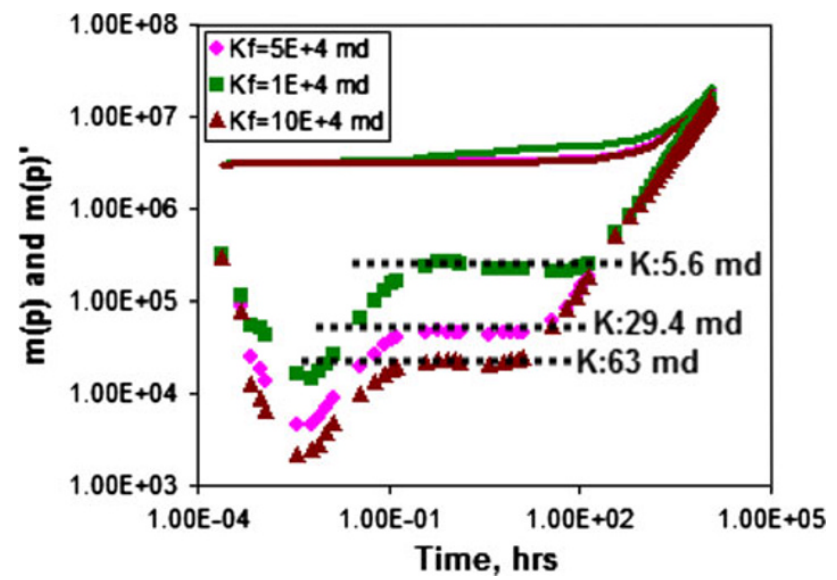

Fig. 6 Effect of fracture permeability $\left(K_{f}\right)$ on welltest permeability

the typical dual-porosity response that is then followed by infinite acting radial flow zero slope line. The late time data are affected by no flow boundary effect, that in the case of higher fracture permeability, its response is reached earlier. Fracture aperture of $0.1,1$ and $10 \mathrm{~mm}$ resulted in welltest

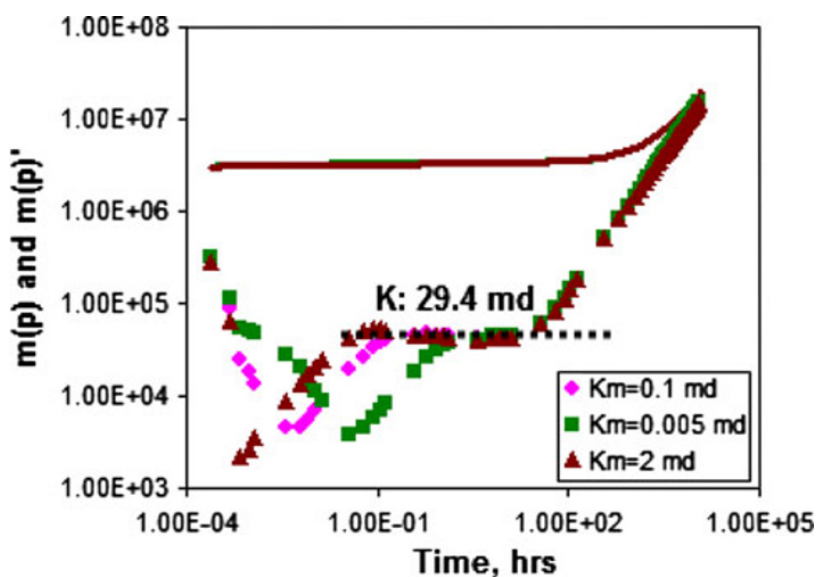

Fig. 7 Effect of matrix permeability $\left(K_{m}\right)$ on welltest permeability

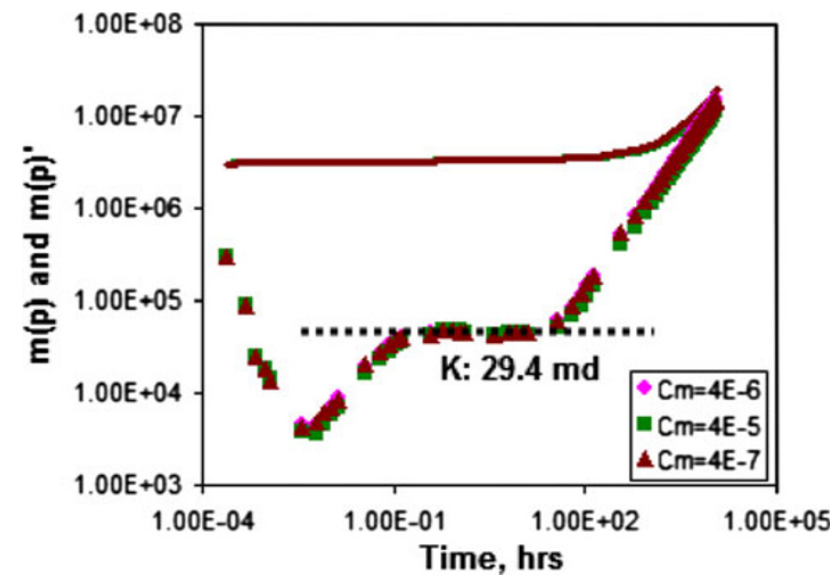

Fig. 8 Effect of matrix compressibility $\left(C_{m}\right)$ on welltest permeability

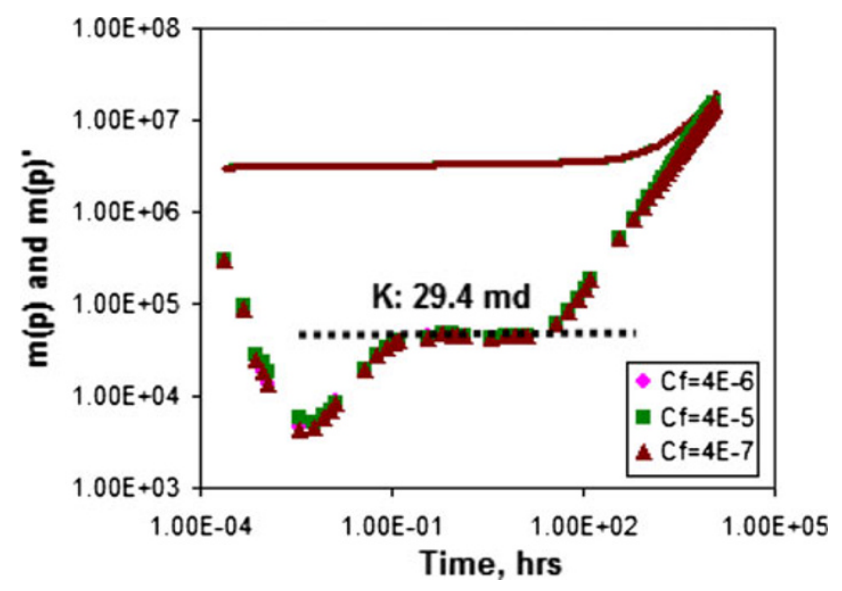

Fig. 9 Effect of fracture compressibility $\left(C_{f}\right)$ on welltest permeability 


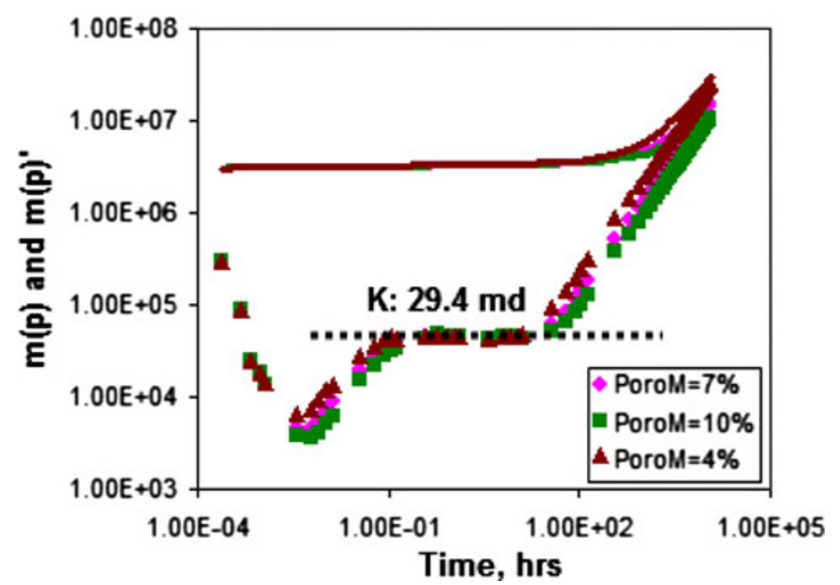

Fig. 10 Effect of matrix porosity $\left(\right.$ Poro $\left._{M}\right)$ on welltest permeability

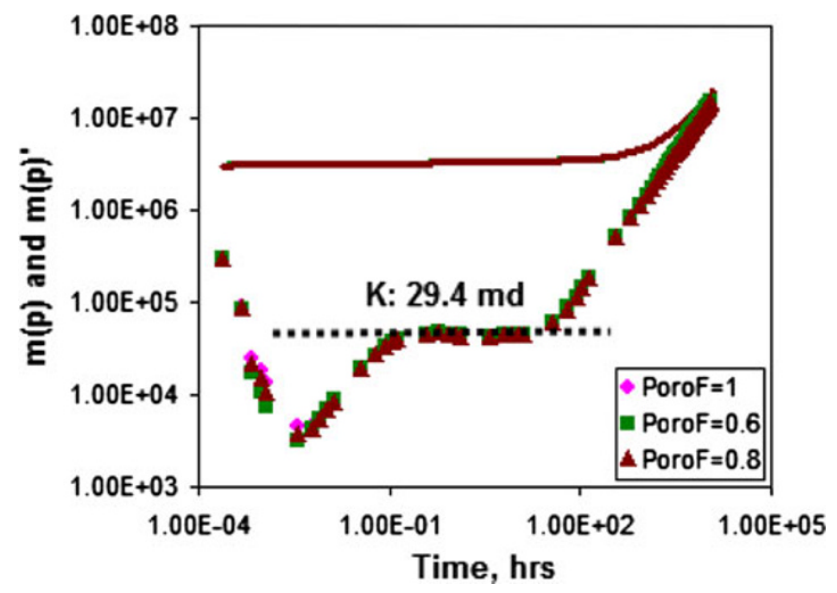

Fig. 11 Effect of fracture porosity $\left(\right.$ Poro $\left._{F}\right)$ on welltest permeability

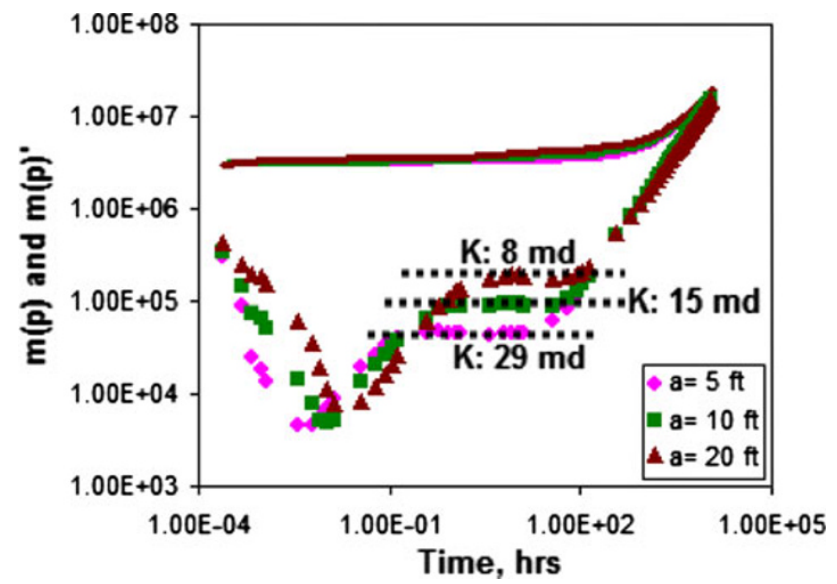

Fig. 12 Effect of fracture spacing $(a)$ on welltest permeability permeability of $2.7,29.4$, and $316 \mathrm{md}$, respectively. Similarly, the effect of fracture permeability, matrix permeability, matrix compressibility, fracture compressibility, matrix porosity, fracture porosity and fracture spacing are shown in Figs. 6, 7, 8, 9, 10, 11 and 12.

Among the parameters examined in the sensitivity analysis, it is observed that only fracture aperture, fracture permeability and matrix block size (fracture spacing) have posed significant impact on welltest permeability, and the effect of other parameters such as matrix permeability, compressibility of matrix and fracture, and porosity of matrix and fracture can be disregarded. Figure 13 shows the relationship between welltest permeability and each of the main fracture parameters. The observations on the reservoir simulation results are in good agreement with the derived Eq. (8):

- Fracture permeability is mainly function of welltest permeability, fracture aperture and fracture spacing.

- Fracture permeability has linear relationship with matrix block size and welltest permeability, and inverse relationship with fracture aperture (i.e., if $K_{f}$ is increased, to match the welltest permeability, $b$ should be reduced).

Combining the curve fitting functions shown in Fig. 13 results in the following equation [Eq. (10)]:

$K_{f}=0.795 * K_{\text {welltest }} *\left(\frac{a}{b}\right)^{1.04}$

where $K_{f}$ is fracture permeability in $\mathrm{md}, b$ is fracture aperture in $\mathrm{ft}$ and $a$ is fracture spacing in $\mathrm{ft}$. The plot of estimated welltest permeability [from Eq. (10)] versus model welltest permeability has been shown in Fig. 14. Comparing the actual simulation outputs and the results from the Eq. (10), it can be observed that the average error is around $5 \%$, indicating that the multi-variable regression results for the constants $C_{1}$ and $C_{2}$ are reliable.

The proposed method [Eq. (10)] is based on Kazemi dual-porosity dual-permeability model that has a layered formation, and therefore this approach may perform reasonably well in the formations with high density low angle fracture network, more specifically in the range of the fracture parameters used in sensitivity analysis. The approach is fairly simple, and deeply rooted in the simplified vision of the fractured rock of the Kazemi model, and may provide good first guess values for fracture parameters.

The estimated fracture parameters can be considered as initial guess in reservoir simulation models for naturally fractured tight gas reservoirs, and then be tuned during history matching to get more reliable results for natural fractures productivity and their contribution on total gas production. 
Fig. 13 Relationship between fracture parameters and welltest permeability

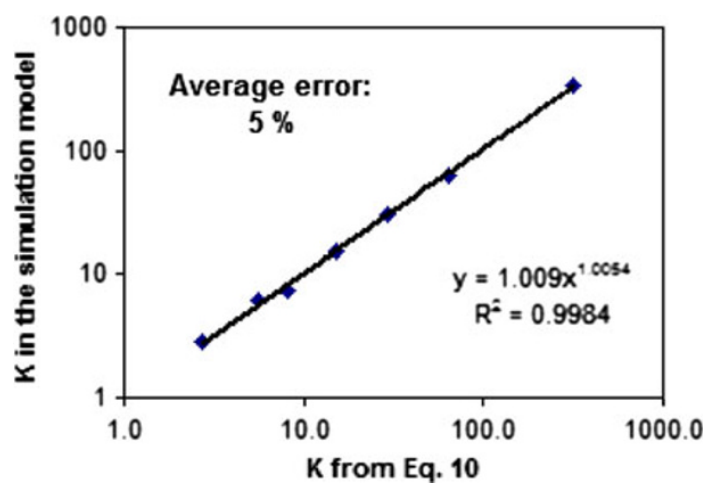

Fig. 14 Welltest permeability from the model, versus welltest permeability calculated from Eq. (10)

\section{Field example: fracture characterization}

For a well completed in a naturally fractured tight gas reservoir (with average matrix permeability of $0.1 \mathrm{md}$ ), results of welltest data, core analysis, and image log in water-based mud processing are studied and integrated to characterize fracture parameters.

Pressure fall-off test was performed in this well by injecting water for a period of time, followed by pressure fall-off test. Pressure transient data analysis results are shown in Fig. 15, in which the results showed average permeability of $75 \mathrm{md}$ for the naturally fractured formation. The Formation Micro Imaging (FMI) Log data acquired after the well drilling using water-based mud was also studied. The results for fracture distribution, fracture

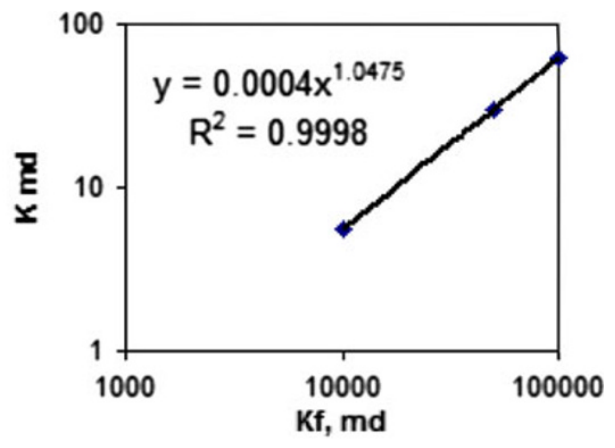

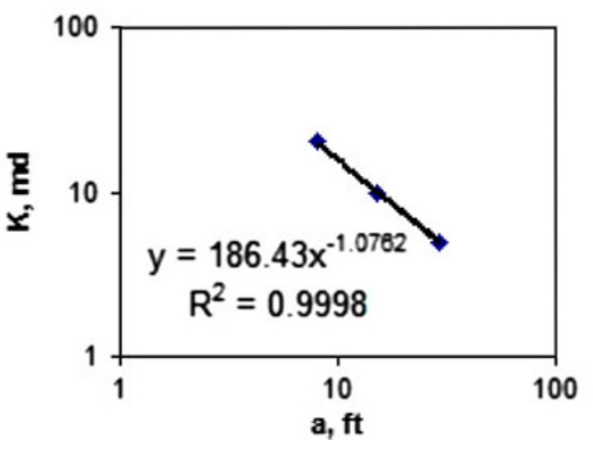

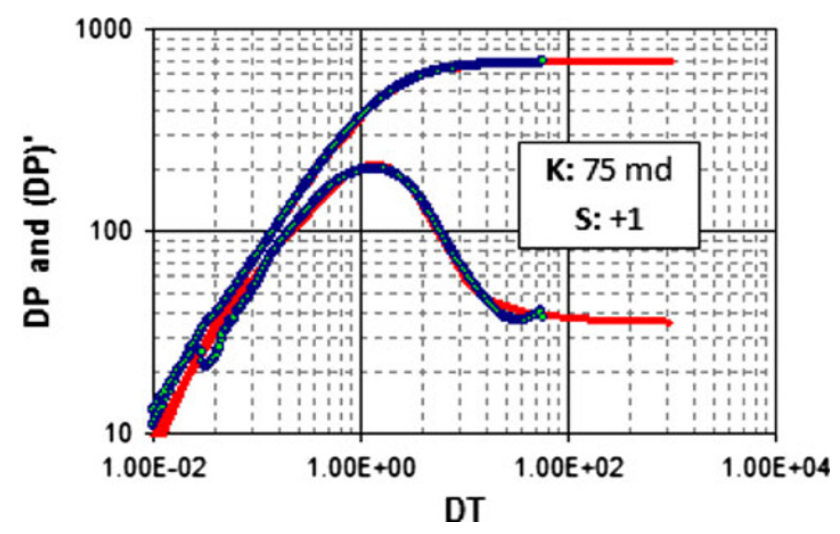

Fig. 15 Pressure transient testing analysis and results

aperture and fracture porosity are shown in Table 3 and Figs. 16, 17 and 18. The data processing results in this well-showed average fracture porosity of $0.3 \%$, average matrix block size of $0.93 \mathrm{ft}$, and average fracture aperture of $0.1 \mathrm{~mm}$. Using welltest permeability and image log fracture spacing and aperture as input data into Eq. (10), it resulted in fracture permeability of $174,000 \mathrm{md}$.

It should be noted that in this case, the image log fracture parameters might be different compared with fracture parameters during the pressure transient testing. Injection of water prior to pressure fall-off test may have increased aperture of natural fractures in the water invaded reservoir zone around wellbore (over estimating actual fracture

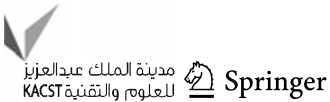


Table 3 Natural fractures data summary

\begin{tabular}{lll}
\hline Image log processing results & & \\
Average fracture density & $1 / \mathrm{ft}$ & 1.04 \\
Number of open fractures $(n)$ & - & 57 \\
Average matrix block size $(a)$ & $\mathrm{ft}$ & 0.97 \\
Average Fracture Aperture $(b)$ & $\mathrm{mm}$ & 0.1 \\
Average Fracture Porosity $(\varphi f)$ & $\%$ & 0.3 \\
Fracture permeability from Eq. $(10)$ & & \\
Estimated fracture permeability $\left(K_{f}\right)$ & $\mathrm{md}$ & 174,000 \\
\hline
\end{tabular}

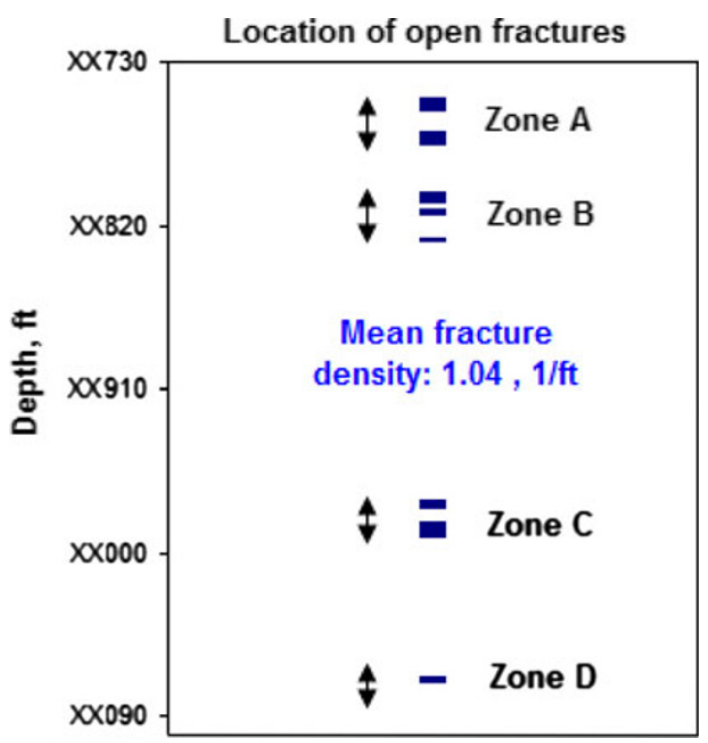

Fig. 16 Fracture distribution data from Image Log processing results (Net reservoir thickness $57 \mathrm{ft}$ )

permeability from welltest data). In the case of pressure drawdown followed by pressure build-up, the results for fracture permeability might be different.

\section{Conclusions}

- Natural fractures in the tight formations make significant contribution on production, and therefore it is essential to estimate their dynamic characteristics.

- In tight formations, due to the weak reservoir response to pressure disturbance, the interporosity flow coefficient and fracture storativity coefficients might not be possible.

- Welltesting analysis in tight gas reservoirs has uncertainties and may not directly provide characterisation of fracture dynamic parameters such as fracture storativity and interporosity flow coefficient.

- Welltest permeability is mainly controlled by fracture permeability, matrix block size and fracture aperture,

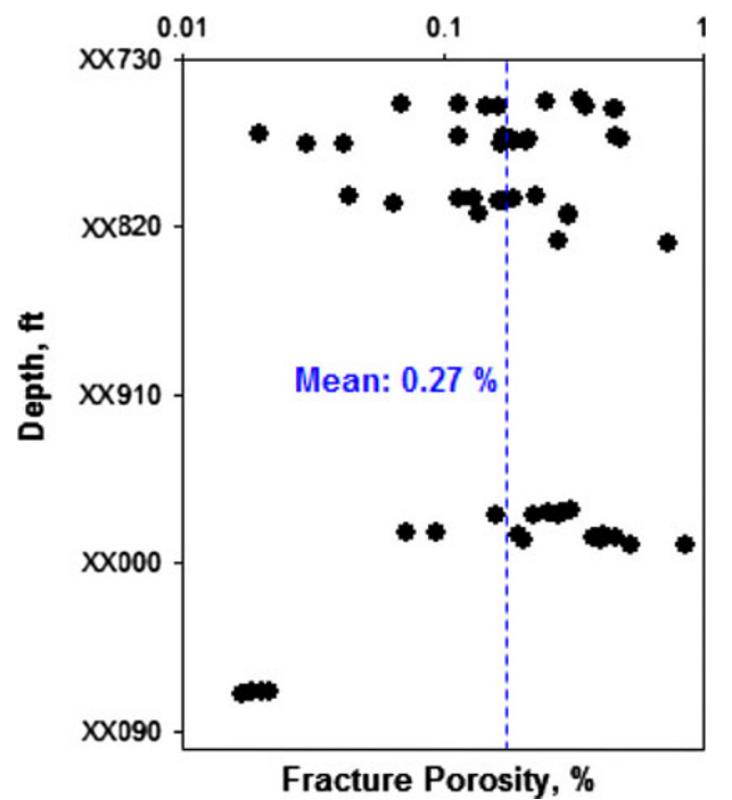

Fig. 17 Fracture porosity data from Image Log processing results

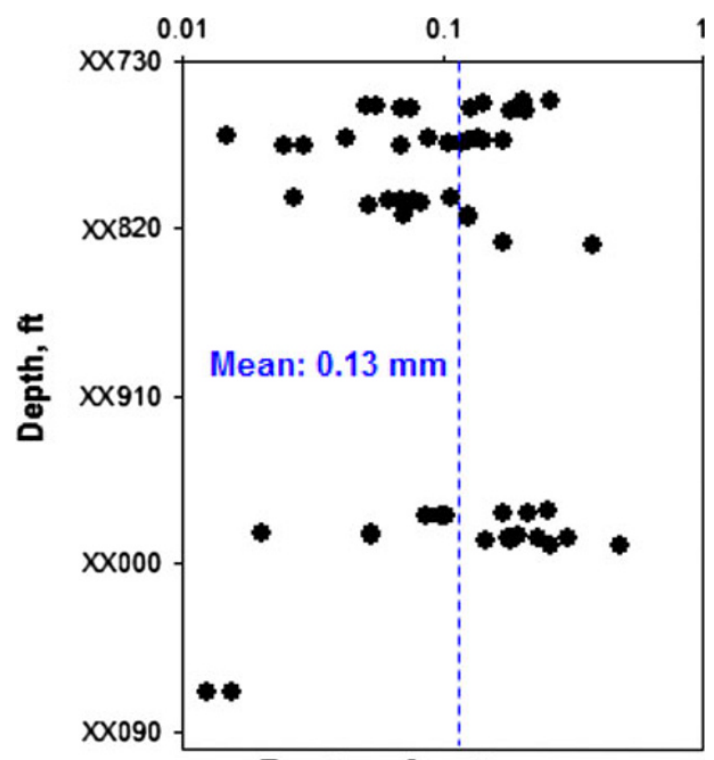

Fracture Aperture, mm

Fig. 18 Fracture aperture data from Image Log Processing Results

and it is not very sensitive to matrix permeability, matrix and fracture compressibilities, and matrix and fracture porosities.

- In addition to petrophysical evaluation, results that provide important input for welltest analysis of conventional reservoirs, image $\log$ data are needed in welltest analysis of naturally fractured tight gas reservoirs. 
- Using welltest permeability and image log fracture spacing and aperture, by considering average permeability based on the thickness of fracture and matrix layers, the proposed method can provide reliable first guess estimation of average fracture permeability for reservoir simulation studies.

Acknowledgments The authors are deeply thankful to Professor Ali Saeidi, the author of the book "Reservoir Engineering of Fractured Reservoirs" for their valuable guides and helpful discussions to initiate this work. We also would like to appreciate Computer Modeling Group (CMG) for use of CMG-IMEX software and Kappa Engineering for use of Kappa-Ecrin software in this study.

Open Access This article is distributed under the terms of the Creative Commons Attribution License which permits any use, distribution, and reproduction in any medium, provided the original author(s) and the source are credited.

\section{References}

Bahrami H, Rezaee R, Kabir A, Siavoshi J, Jammazi R (2010) Using second derivative of transient pressure in welltest analysis of low permeability gas reservoirs, SPE 132475. Production and Operations Conference and Exhibition, Tunis, Tunisia
Bourdarot G (1998) Well Testing Interpretation Methods, Editions Technip

Dashti R, Bagheri MB (2009) Fracture characterization of a porous fractured carbonate reservoir, SPE 125329. SPE reservoir characterization conference, UAE

Garcia JP, Pooladi-Darvish M, Brunner F, Santo M, Mattar L (2006) Welltesting of tight gas reservoirs, SPE 100576. SPE Gas Technology Symposium, Calgary

Kazemi H, Merrill LS, Porterfield KL, Zeman PR (1976) Numerical Simulation of Water-Oil Flow in Naturally Fractured Reservoirs, SPEJ (Dec. 1976) 317-326

Luthi SM (1990) Fracture apertures from electrical borehole scans, J Geophys 55 (NO.7)

Odeh AS (1981) Comparison of solutions to a three-dimensional black-oil reservoir simulation problem, SPE 9723. J Petroleum Technol (JPT) 13-25

Racht TD, Van Golf (1982) Fundamentals of fractured reservoir engineering, 1st edn, Elsevier

Reiss LH (1980) The reservoir engineering aspects of fractured formation, Editions TECHNIP, Paris

Restrepo DP, Tiab D (2009) Multiple Fractures Transient Response, SPE 121594. Latin American and Caribbean Petroleum Engineering Conference, Cartagena

Saeidi Ali M (1987) Reservoir engineering of fractured reservoirs, Total, Paris

Tiab D, Restrepo DP, Lgbokoyi A (2006) Fracture porosity of naturally fractured reservoirs, SPE 104056. First International Oil Conference and Exhibition in Mexico, Mexico 